\title{
Optical scattering field imaging along a nanofiber in operando
}

\author{
Yosri Haddad *, Jacques Chrétien, Samuel Margueron, Jean-Charles Beugnot, \& Gil Fanjoux \\ FEMTO-ST Institute, optics department, UMR 6174 CNRS / Université Bourgogne Franche-Comté, 25030 Besançon - France
}

\begin{abstract}
We present a non-destructive and non-invasive imaging spectroscopic technique with a high spatial and spectral resolution based on the detection of the Rayleigh scattering field radiated out of a centimetric length and micrometric size optical waveguide in operation.
\end{abstract}

\section{Introduction}

The linear or nonlinear optical propagation behaviour lengthwise of micro- and nano- waveguides in operation is difficult to experimentally evaluate, although this information is crucial for their optimal development. Previously, apart from the destructive cut-back method, far-field qualitative imaging of Rayleigh scattering (RS) have been used for rapid analysis of the propagation of light in few-mode optical nanofibers (ONF) [1]. In the present work, we demonstrate a novel method, nondestructive and non-invasive, with a high spatial and spectral resolution, to analyse the light propagation in an optical waveguide in operation. By analysing with a confocal spectrometer the RS radiated out of an ONF in operation, we show that our method can spatially characterize the entire ONF in the linear regime with a micrometric spatial resolution. This approach provides direct observation of the higher-order modes (HOMs) leaking from the ONF surface along the taper region and the transition from core-cladding guidance to claddingair guidance. This method can also be used to characterize the linear loss and provides the localization of defects along an optical waveguide.

\section{Experimental Setup}

Our measurement setup, illustrated in Fig. 1, consists of a high-precision motorization for longitudinal $\mathrm{X}$ and transverse $\mathrm{Y}$ and $\mathrm{Z}$ translation (with a step size $<10 \mathrm{~nm}$ ) of the component under study, allowing the generation of an accurate recordable 3D spatial trajectory point-bypoint. RS of the guided light emitted from the outside of an optical waveguide is collected in the direction orthogonal to the propagation direction (Fig. 1) with a $\times 10$ microscope objective $(\mathrm{NA}=0.3)$ and recorded through a confocal spectrometer. The spectrometer monochromator is equipped with three distinct diffraction gratings (300, 1500 and 2400 lines $/ \mathrm{mm}$ ), and an ultra-sensitive cooled $\mathrm{CCD}$ detector $\left(-80^{\circ} \mathrm{C}\right)$ to have a low detection threshold. Alternatively, a CCD image camera is used to image the waveguide for positioning. The waveguide studied in this work corresponds to an ONF fabricated from telecom single mode fiber (SMF@1550nm) as described in [2]. After tapering process, the ONF has a uniform waist of $1 \mu \mathrm{m}$ over around $17 \mathrm{~mm}$ accurately measured by Brillouin scattering methods [2], and the two transition tapers are around $37 \mathrm{~mm}$ long (Fig. 1). The laser beam injected in the ONF comes from a wavelength and power stabilized $\mathrm{cw}$ laser diode with a wavelength of $653 \mathrm{~nm}$. The fiber before stretching is therefore strongly multimode at this wavelength. The ONF is then placed inside a closed box to protect it from external air vibrations and to reduce any kind of contamination. Moreover, we monitored the transmitted power during the RS measurement and we did not observe any significant variation (Fig. 1).

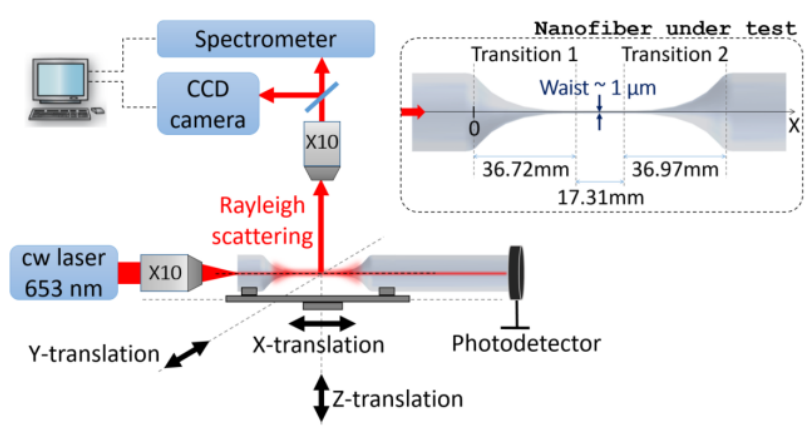

Fig. 1. Schematic experimental setup, and geometrical data of the ONF studied in this work (in dashed box).

\section{Experimental results and discussion}

First, CCD camera pictures of the fiber allow the measurement of the evolution of the fiber taper radius with the distance $X$ (black crosses in Fig. 2a). This analyzed fiber part includes the end of the non-stretched SMF fiber $(62.5 \mu \mathrm{m})$, the taper region, and the ONF.

Fig. $2 b$ shows an XY-2D mapping in logarithm scale of the RS intensity along the fiber input side before the ONF. Note that no normalization in intensity has been realized. Therefore, the evolution of the RS intensity directly represents the evolution of the scattering process. For the first two millimeters, Fig. 2 b shows that the light is only confined in the core of the fiber and thus the fluctuations in refractive index of the core dominate the scattering process. The RS signal shows a uniform trace and the

* Corresponding author: yosri.haddad@femto-st.fr 

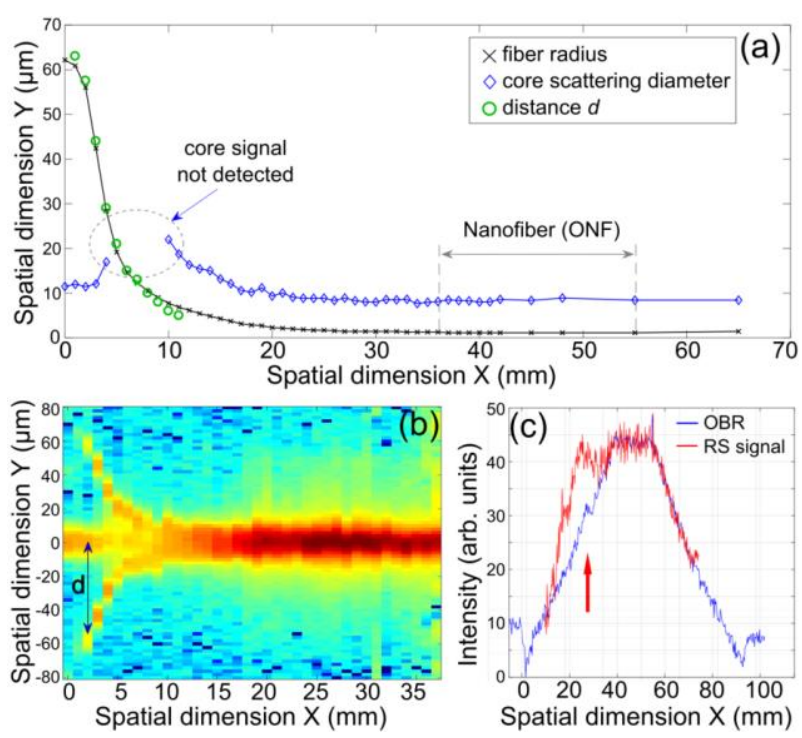

Fig. 2. (a) Evolution vs. the longitudinal distance $X$ of the fiber radius $(\mathbf{X})$, the linewidth (FWHM) of the scattering signal from the core $(\diamond)$, and the distance $d$ between the scattering traces symmetrically shifted from the core (O). (b) 2D XY mapping of RS intensity in the transition 1 (logarithm scale). (c) Evolution of RS intensity from OBR trace (blue) and our measurements (red).

linewidth of the core signal is almost stable (blue diamond in Fig. 2a). After $2 \mathrm{~mm}$, the linewidth clearly increases meaning that light progressively escapes the core to the cladding along the taper. Therefore, a substantial portion of the optical power is propagating within the cladding. In addition, Fig. $2 \mathrm{~b}$ shows that the scattering light presents three distinct traces, one central peak corresponding to the core, and two scattering traces symmetrical with respect to the core by a distance $d$. In fig. 2 a, the green circles show the evolution of the distance $d$ along X. These match clearly the evolution of the radius in the taper region. This mean that these two scattering traces originate from the surface. Thereafter, between $5 \mathrm{~mm}$ and $10 \mathrm{~mm}$, as the fiber core signal is negligible, the central scattering peak is no more measurable because of its superposition with the two lateral peaks. This shows that the amount of light remaining in the core is now extended into the cladding. Here, the refractive-index fluctuations of the cladding dominate the scattering process. Finally, after $10 \mathrm{~mm}$, the RS signal show a single trace again which increases in intensity with the propagation distance as it approaches the nanofiber as we will explain thereafter. In this region, the surrounding air becomes the cladding and the whole fiber the core. Moreover, the taper surface roughness dominates the scattering process [3]. On the other hand, the signal linewidth decreases up to a limit of about $8 \mu \mathrm{m}$. As the nanofiber has a waist diameter equal to $1 \mu \mathrm{m}$, this threshold corresponds to the spatial resolution of our system. This resolution can reach $5 \mu \mathrm{m}$ by reducing the width of the entrance slit of the spectrometer to $10 \mu \mathrm{m}$. Let us analyze the evolution of the longitudinal RS signal intensity with the distance $X$. We have therefore compared our experimental results with the state-of-art method, i.e., an OBR system from Luna ${ }^{\circledR}$ which uses an internal $1566 \mathrm{~nm}$ laser source. Fig. 2c shows the OBR trace (blue curve) and our scattering trace (red curve). Fig. 2c shows that the scattering signal shape obtained with our technique with a wavelength of $653 \mathrm{~nm}$ is in good agreement with the OBR signal shape. Nevertheless, our signal in transition 1 is strongly blurred by a large increase (red arrow), contrary to the RS signal in transition 2. As the fiber is multimode and the tapered transition is not adiabatic, coupling of guided modes with lossy modes induces the spatially filtering by the transition of several high order modes. Therefore, the lossy modes cross the interface glass-air and consequently undergo large scattering by surface roughness.

To confirm this observation, we have analyzed an ONF with $1 \mu \mathrm{m}$ of diameter and $2 \mathrm{~cm}$ long, fabricated from a single mode fiber in the visible range SM450. Fig. 3 shows an XY mapping RS intensity (in logarithm scale) for this ONF under the same conditions as Fig 2b. The RS intensity shows the absence of the surface scattering in the taper region (transition 1). This confirms that the surface scattering obtained for the multimode ONF (Fig. 2) come from the no longer guided HOMs modes, which go out the fiber in the transition 1. Accordingly, when reach cutoff, those modes cross the surface of the fiber presenting a high scattering efficiency. Fig. 3 also clearly shows that the guiding process drastically change near $\mathrm{X}=10 \mathrm{~mm}$ of propagation, from a core-cladding to a fiber-air guidance, in perfect agreement with numerical prediction.

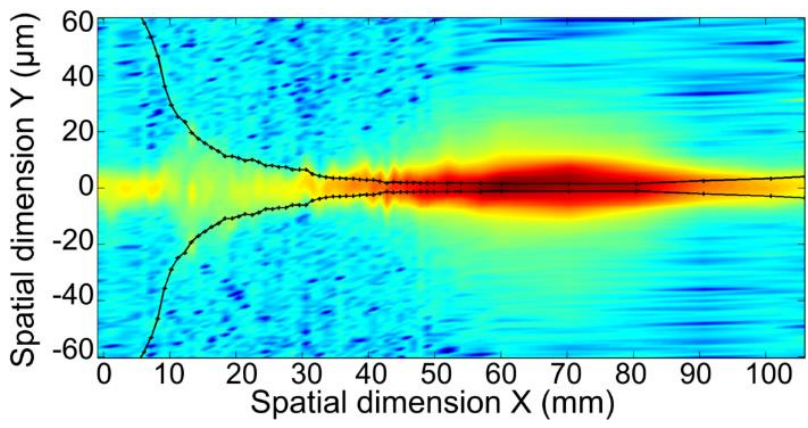

Fig. 3. 2D XY mapping of RS intensity for a single mode fiber (a)653nm with adiabatic transition (logarithm scale). Black curves correspond to the fiber diameter from CCD camera.

\section{Conclusion}

Preliminary results have shown that our technique is a powerful tool to characterize the evolution of the field propagating along a photonic waveguide with a micrometric spatial resolution. For a nanofiber, RS signal provides direct observation of the transition from corecladding to cladding-air guidance. Moreover, we have shown that the presence of the surface scattering indicates non-adiabaticity in the tapering part.

This work has been supported by the EIPHI Graduate School (contract ANR-17-EURE-0002) and by the Conseil Régional de Bourgogne - Franche-Comté (DECAP and CIPPIC project).

\section{References}

1. J. E. Hoffman et al., Optica 2, 416-423 (2015).

2. A. Godet et al., Optica 4, 1232-1238 (2017).

3. Y.-H. Lai et al., Opt. Exp. 25, 22312 (2017). 